\title{
OPTIMAL SYSTEM FOR SUM OF TWO IDEALS ADMITTED BY HYDRODYNAMIC TYPE EQUATIONS
}

\author{
S.V. KHABIROV
}

\begin{abstract}
We introduce the rules for constructing the optimal system of the dissimilar subalgebras for the sum of two ideals for which the optimal systems are known. As a result, we give the dissimilar subalgebra for five not yet considered Lie algebra admitted by the hydrodynamic type equations. It completes the listing of the subalgebras for the Lie algebras in the group classification of the gas dynamic models by the state equation.
\end{abstract}

Keywords: hydrodynamic type equations, Lie algebra, optimal system of subalgebras.

Mathematics Subject Classification: 35B06, 35Q35

\section{INTRODUCTION}

In work [1] there was initiated program PODMODELI for gas dynamics equations. The main goals of the program are calculation of admitted group (Lie algebra), group classification by the state equation, construction of optimal system of subalgebras for the models of group classification, study of submodels constructed by subalgebras. It was show in work [2] that there exist 10 non-isomorphic finite-dimensional Lie algebras for the models of hydrodynamical type. It remained to constructed optimal systems for five Lie algebras of sum of two ideals for which the optimal systems were constructed. All the models of hydrodynamical type admit 11-dimensional Lie algebra $L_{11}$ with the basis in the Cartesian coordinate systems formed by the translations along the space $T_{3}=\left\{X_{1}=\partial_{x}, X_{2}=\partial_{y}, X_{3}=\partial_{z}\right\}$, Galilean translations $\Gamma_{3}=\left\{X_{4}=t \partial_{x}+\partial_{u}, X_{5}=\partial_{y}+\partial_{v}, X_{6}=\partial_{z}+\partial_{w}\right\}$, rotations $S_{3}=\left\{X_{7}=z \partial_{y}-y \partial_{z}+w \partial_{v}-v \partial_{w}\right.$, $\left.X_{8}=x \partial_{z}-z \partial_{x}+u \partial_{w}-w \partial_{u}, X_{9}=y \partial_{x}-x \partial_{y}+v \partial_{u}-u \partial_{v}\right\}$, time translations $X_{10}=\partial_{t}$, and uniform dilatation $X_{11}=t \partial_{t}+x \partial_{x}+y \partial_{y}+z \partial_{z}$ [1]. The optimal system for algebra $L_{11}$ was constructed in work [1]. The model of gas dynamics with the state equation, where the density is a product, admits Lie algebra $L_{12}=\left\{X_{12}\right\} \dot{\oplus} L_{11}$ (a semi-direct sum of subalgebra and ideal), where $X_{12}=t \partial_{t}-u \partial_{u}-v \partial_{v}-w \partial_{w}+2 \rho \partial_{\rho}$ is one more dilatation. The optimal system for $L_{12}$ was constructed in work [3]. The model with zero sound speed admits infinite-dimensional Lie algebra having the maximal finite-dimensional Lie algebra $L_{12} \oplus P_{3}$ (the direct sum of two ideals), where $P_{3}=\left\{Y_{1}=\partial_{p}, Y_{p}=\rho \partial_{\rho}+p \partial_{p}, Y_{p^{2}}=2 \rho p \partial_{\rho}+p^{2} \partial_{p}\right\}$ is a simple algebra non isomorphic to $S_{3}$ over the field of real numbers $R$. There are just two nonsimilar subalgebras in $P_{3}$, these are $\left\{Y_{1}\right\},\left\{Y_{1}, Y_{p}\right\}$ [2]. The optimal system for $L_{11} \oplus\left\{Y_{1}\right\}$ was constructed in [4]. In the present work we shall describe optimal systems for Lie algebras each being a sum of two ideals: $L_{11} \oplus\left\{Y_{1}, Y_{p}\right\}, L_{11} \oplus\left\{Y_{1}, Y_{p}, Y_{p^{2}}\right\}, L_{12} \oplus\left\{Y_{1}\right\}, L_{12} \oplus\left\{Y_{1}, Y_{p}\right\}, L_{12} \oplus\left\{Y_{1}, Y_{p}, Y_{p^{2}}\right\}$. The structure constants of simple algebra $P_{3}$ are defined by the commutators: $\left[Y_{1}, Y_{p}\right]=Y_{1}$, $\left[Y_{1}, Y_{p^{2}}\right]=2 Y_{p},\left[Y_{p}, Y_{p^{2}}\right]=Y_{p^{2}}$, while for algebra $S_{3}$ the same is done by the commutators:

S.V. Khabirov, Optimal System for sum of two ideals admitted By HYDrodynamic type EQUaTIONS.

(C) KHABiRov S.V. 2014 .

The work is supported by RFBR (12-01-00648, 14-01-97027), the Grant of the President of Russia for supporting leading scientific schools (no. NSh-6706.2012.1), the grant No.11.G34.31.0042 of the government of Russia under the decree no. 220.

Submitted December 5, 2013. 
$\left[X_{7}, X_{8}\right]=X_{9},\left[X_{8}, X_{9}\right]=X_{7},\left[X_{9}, X_{7}\right]=X_{8}$. There are just two simple three-dimensional Lie algebras nonisomorphic over field $R$.

The description of the procedure of representing the optimal system for a sum of two ideals is based on the constructed optimal systems of each ideal. In each ideal there act its automorphisms. The commutators of basis operators of subalgebras single out subalgebras in the ideal which are already reduced by internal automorphisms to subalgebras in the optimal system of the ideal. This is why in what follows the internal automorphisms are not used and we do not provide them here. The found subalgebras possess special properties, and by means of the latter they are sought in the optimal system of the ideal. It is sufficient to list these subalgebras in order to construct optimal system for the sum of two ideals by certain rules.

\section{Optimal System FOR Lie Algebra $L_{11} \oplus\left\{Y_{1}, Y_{p}, Y_{p^{2}}\right\}$}

Each subalgebra of dimension $k+1$ in the algebra $L_{11} \oplus\left\{Y_{1}\right\}$ is represented by the basis $Z_{1}=X_{i_{1}}+\ldots, Z_{2}=X_{i_{2}}+\ldots, \ldots, Z_{k}=X_{i_{k}}+\ldots, Y_{1}+Z_{k+1}$, where $Z_{k+1}$ and the dots after the plus sign stand for a linear combination of basis elements in $L_{11}$ not containing $X_{i_{1}}, \ldots, X_{i_{k}}$. The linear sets $\left\{Z_{1}, \ldots, Z_{k}\right\},\left\{Z_{1}, \ldots, Z_{k}, Z_{k+1}\right\}$ are subalgebras in $L_{11}$. The subalgebra $\left\{Z_{1}, \ldots, Z_{k}\right\}$ is an ideal in $\left\{Z_{1}, \ldots, Z_{k}, Z_{k+1}\right\}$. It is implied by the fact that operator $Y_{1}$ commutes with each element in $L_{11}$. The listing rule for subalgebras in $L_{11} \oplus\left\{Y_{1}\right\}$ is as follows: for each subalgebra in $L_{11}$ one should find a subalgebra with the dimension greater by one for which this subalgebra is an ideal. All such subalgebras are listed in [4]. If we add $Y_{1}$ to a basis of $L_{11}$, we obtain the trivial extension. Trivial extensions were not reduced in the optimal system of work [4].

1.1. Optimal system for Lie algebra $L_{11} \oplus\left\{Y_{1}, Y_{p}\right\}$. Each subalgebra of dimension $k+2$ in algebra $L_{11} \oplus\left\{Y_{1}, Y_{p}\right\}$ is represented by a basis of the above introduced operators $Z_{1}, \ldots, Z_{k}$, $Y_{1}+Z_{k+1}$ and operator $Y_{p}+Z_{k+2}$, where $Z_{k+2}$ is a linear combination of basis elements $L_{11}$ not involving $X_{i_{1}}, \ldots, X_{i_{k}}$. Linear spans $\left\{Z_{1}, \ldots, Z_{k}\right\},\left\{Z_{1}, \ldots, Z_{k}, Z_{k+1}\right\},\left\{Z_{1}, \ldots, Z_{k}, Z_{k+2}\right\}$ are subalgebras in $L_{11}$. Subalgebra $\left\{Z_{1}, \ldots, Z_{k}\right\}$ is an ideal in $\left\{Z_{1}, \ldots, Z_{k}, Z_{k+1}\right\}$ and in $\left\{Z_{1}, \ldots, Z_{k}, Z_{k+2}\right\}$. Linear spans $\left\{Z_{1}, \ldots, Z_{k}, Y_{1}+Z_{k+1}\right\},\left\{Z_{1}, \ldots, Z_{k}, Y_{p}+Z_{k+2}\right\}$ are subalgebras of optimal system for algebra $L_{11} \oplus\left\{Y_{1}\right\}$. The identity

$$
\left[Y_{1}+Z_{k+1}, Y_{p}+Z_{k+2}\right]=Y_{1}+\left[Z_{k+1}, Z_{k+2}\right]
$$

implies the relation

$$
\left[Z_{k+1}, Z_{k+2}\right]=Z_{k+1}+\sum_{l=1}^{k} C_{12}^{l} Z_{k}
$$

or $Z_{k+1}=0$ and $C_{12}^{l}=0$.

In the latter case one should take a subalgebra in $L_{11} \oplus\left\{Y_{1}\right\}$. There instead of $Y_{1}$ one should write $Y_{p}$ and add operator $Y_{1}$ to the obtained basis. Such subalgebras we also regard as trivial extensions.

In the former case the rule of finding subalgebras is as follows: find two subalgebras in $L_{11} \oplus\left\{Y_{1}\right\}$ reading as $L_{k} \oplus\left\{Y_{1}+Z_{k+1}\right\}, L_{k} \oplus\left\{Y_{1}+Z_{k+2}\right\}$, check identity (1.1), add $L_{k} \oplus\left\{Y_{1}+\right.$ $\left.Z_{k+1}, Y_{p}+Z_{k+2}\right\}$ in the optimal system of the Lie algebra $L_{11} \oplus\left\{Y_{1}, Y_{p}\right\}$. Looking through the optimal system in [4] gives the following result: operator $Y_{p}$ is added to the operator involving $X_{11}$. Operator $Y_{1}$ is added to an operator in the subalgebras of translations $T_{3}$ or to operator $X_{10}$. In total we get 43 subalgebras, 18 of which involve operator $Y_{1}+X_{10}$. It makes no sense to list since they can be easily seen in Table of work [4].

1.2. Optimal system of Lie algebra $L_{11} \oplus\left\{Y_{1}, Y_{p}, Y_{p}^{2}\right\}$. Each subalgebra in algebra $L_{11} \oplus\left\{Y_{1}, Y_{p}, Y_{p^{2}}\right\}$ with a three-dimensional projection on $P_{3}$ is represented by the basis by above introduced operators $Z_{1}, \ldots, Z_{k}, Y_{1}+Z_{k+1}, Y_{p}+Z_{k+2}$ and the operator $Y_{p^{2}}+Z_{k+3}$, where $Z_{k+3}$ is a linear combination of basis elements in $L_{11}$ not involving $X_{i_{1}}, \ldots, X_{i_{k}}$. Linear spans 
$\left\{Z_{1}, \ldots, Z_{k}\right\},\left\{Z_{1}, \ldots, Z_{k}, Z_{k+1}\right\},\left\{Z_{1}, \ldots, Z_{k}, Z_{k+2}\right\},\left\{Z_{1}, \ldots, Z_{k}, Z_{k+3}\right\}$ are subalgebras $L_{11}$. The first subalgebra is an ideal in other subalgebras. The commutators

$$
\begin{aligned}
& {\left[Y_{1}+Z_{k+1}, Y_{p}+Z_{k+2}\right]=Y_{1}+\left[Z_{k+1}, Z_{k+2}\right],} \\
& {\left[Y_{1}+Z_{k+1}, Y_{p^{2}}+Z_{k+3}\right]=2 Y_{p}+\left[Z_{k+1}, Z_{k+3}\right],} \\
& {\left[Y_{p}+Z_{k+2}, Y_{p^{2}}+Z_{k+3}\right]=Y_{p^{2}}+\left[Z_{k+2}, Z_{k+3}\right]}
\end{aligned}
$$

determine the identities

$$
\begin{aligned}
& {\left[Z_{k+1}, Z_{k+2}\right]=Z_{k+1}+\sum_{l=1}^{k} C_{12}^{l} Z_{l},} \\
& {\left[Z_{k+1}, Z_{k+3}\right]=2 Z_{k+2}+\sum_{l=1}^{k} C_{13}^{l} Z_{l},} \\
& {\left[Z_{k+2}, Z_{k+3}\right]=Z_{k+3}+\sum_{l=1}^{k} C_{23}^{l} Z_{l},}
\end{aligned}
$$

with some constants $C_{i j}^{l}$. These identities show that the linear span $\left\{Z_{1}, \ldots, Z_{k}, Z_{k+1}, Z_{k+2}, Z_{k+3}\right\}$ is a subalgebra in $L_{11}$. The projection of this subalgebra on the subspace $\left\{Z_{k+1}, Z_{k+2}, Z_{k+3}\right\}$ is simple algebra $P_{3}$. It is isomorphic to some subalgebra in $L_{11}$. But in $L_{11}$ there is just one simple subalgebra $S_{3}$ which is not similar to $P_{3}$ over field $R$, the contradiction. Thus, $Z_{k+1}=Z_{k+2}=Z_{k+3}=0$ and we obtain only trivial extensions of each subalgebras in $L_{11}$.

\section{Optimal System for Lie Algebra $L_{12} \oplus\left\{Y_{1}, Y_{p}, Y_{p^{2}}\right\}$}

The optimal system for $L_{12}$ was constructed in work [3]. Nonzero commutators of operators $X_{12}, X_{11}$ are $\left[X_{12}, X_{k}\right]=X_{k}, k=4,5,6,\left[X_{12}, X_{10}\right]=-X_{10},\left[X_{11}, X_{l}\right]=-X_{l}, l=1,2,3$, $\left[X_{11}, X_{10}\right]=-X_{10}$. We shall not make use of other commutators.

2.1. Optimal system for Lie algebra $L_{12} \oplus\left\{Y_{1}\right\}$. Each subalgebra of dimension $k+1$ in algebra $L_{12} \oplus\left\{Y_{1}\right\}$ is represented by the basis of the above introduced operators $Z_{1}, Z_{2}, \ldots, Z_{k}$ and the operator $Y_{1}+X_{12}+Z_{k+1}$ or of the above introduced operators $Z_{1}, \ldots, Z_{k-1}$ and the operators $Z_{k}+X_{12}, Y_{1}+Z_{k+1}$, where $Z_{k+1}, Z_{k}$ is a linear combination of basis elements in $L_{11}$ not involving $X_{i_{1}}, \ldots, X_{i_{k}}$. In the first case the linear span $\left\{Z_{1}, \ldots, Z_{k}\right\}$ is a subalgebra in $L_{11},\left\{Z_{1}, \ldots, Z_{k}, X_{12}+Z_{k+1}\right\}$ are subalgebras in $L_{12}$, subalgebra $\left\{Z_{1}, \ldots, Z_{k}\right\}$ is an ideal in $\left\{Z_{1}, \ldots, Z_{k}, X_{12}+Z_{k+1}\right\}$. The rule of finding the subalgebras in $L_{12} \oplus\left\{Y_{1}\right\}$ reads as: subalgebra in $L_{12}$ should have subalgebras $L_{11}$ of the dimension less by one. It is to check that all the subalgebras in the optimal system of $L_{12}$ possess this property. Hence, it is sufficient to add operator $Y_{1}$ to the operator involving $X_{12}$ (it is present only in one of the basis operator).

In the second case the rule of finding subalgebras in $L_{12} \oplus\left\{Y_{1}\right\}$ is as follows: $\left\{Z_{1}, \ldots, Z_{k-1}, Z_{k+1}\right\}$ is a subalgebra in $L_{11},\left\{Z_{1}, \ldots, Z_{k-1}, X_{12}+Z_{k}, Z_{k+1}\right\},\left\{Z_{1}, \ldots, Z_{k-1}, X_{12}+Z_{k}\right\}$, $\left\{Z_{1}, \ldots, Z_{k-1}, Z_{k}, Z_{k+1}\right\}$ are subalgebras in $L_{12} ;\left\{Z_{1}, \ldots, Z_{k-1}\right\}$ is an ideal in $\left\{Z_{1}, \ldots, Z_{k-1}, Z_{k+1}\right\}$ and in $\left\{Z_{1}, \ldots, Z_{k-1}, Z_{k}\right\} ;\left[Z_{k}, Z_{k+1}\right]$ is a linear combination of other operators. It is clear that a subalgebra in $L_{12}$ satisfies this property if the operator not involving $X_{12}$ commutes with the other; to one of them we add $Y_{1}$. such subalgebras are regarded as trivial extension. All two-dimensional subalgebras are of this type. For each non-abelian subalgebras in $L_{12}$ of dimension at least three we choose a basis operator not involving $X_{12}$ so that other operators form an ideal. To this operator not commuting with the others, we add $Y_{1}$. Then we check the subalgebra condition. Looking through the optimal system in [3], we arrive at the following result. Operator $Y_{1}$ is added to the operator involving either $X_{7}$ or $X_{11}$ or $X_{10}$ if in the operator containing $X_{12}$ involves a combination $X_{12}-X_{11}$. At that, one can not add anything to the 
operators of rotations subalgebra. The subalgebras are easily written by Table in work [3] and we do not provide them here because of the size reason.

2.2. Optimal system for the Lie algebra $L_{12} \oplus\left\{Y_{1}, Y_{p}\right\}$. Each subalgebra of dimension $k+2$ in the algebra $L_{12} \oplus\left\{Y_{1}, Y_{p}\right\}$ can be represented by the basis of the above introduced operators $Z_{1}, \ldots, Z_{k}, Y_{1}+Z_{k+1}$ and the operator $Y_{p}+X_{12}+Z_{k+2}$ or of above introduced operators $Z_{1}, \ldots, Z_{k-1}, Z_{k}+X_{12}, Y_{1}+Z_{k+1}, Y_{p}+Z_{k+2}$. In the first case the linear span $\left\{Z_{1}, \ldots, Z_{k}\right\}$ is an ideal in the subalgebras $\left\{Z_{1}, \ldots, Z_{k}, Z_{k+1}\right\} \subset L_{11},\left\{Z_{1}, \ldots, Z_{k}, X_{12}+Z_{k+2}\right\} \subset L_{12}$ and the identity

$$
\left[Z_{k+1}, X_{12}+Z_{k+2}\right]=Z_{k+1}+\sum_{l=0}^{k} D^{l} Z_{l}
$$

holds true. It follows that $\left\{Z_{1}, \ldots, Z_{k}, Z_{k+1}, X_{12}+Z_{k+2}\right\}$ is a subalgebra in $L_{12}$. If $Z_{k+1}=0$, then $D^{l}=0$ and for each subalgebra of the algebras $L_{12} \oplus\left\{Y_{1}\right\}$ one should write $Y_{p}$ and add $Y_{1}$ instead of $Y_{1}$. We obtain the trivial subalgebra in $L_{12} \oplus\left\{Y_{1}, Y_{p}\right\}$. If $Z_{k+1} \neq 0$, for each subalgebra in $L_{12} \oplus\left\{Y_{p}\right\}$ one should choose the operator not commuting with $X_{12}+Z_{k+2}$, while the others should form an ideal, and then one should check identity (2.1). Looking through the optimal system, we see that it is possible only for the subalgebras with basis operators $X_{10}$ to which operator $Y_{1}$ is added and there is no operator $X_{11}$ in other basis operators.

In the second case the linear span $\left\{Z_{1}, \ldots, Z_{k-1}\right\}$ is an ideal of subalgebra $\left\{Z_{1}, \ldots, Z_{k-1}, X_{12}+\right.$ $\left.Z_{k}, Z_{k+1}, Z_{k+2}\right\} \subset L_{12}$. The identities

$$
\begin{gathered}
{\left[Z_{k+1}, X_{12}+Z_{k}\right]=\sum_{l=0}^{k-1} A^{l} Z_{l}, \quad\left[Z_{k+2}, X_{12}+Z_{k}\right]=\sum_{l=0}^{k-1} B^{l} Z_{l}} \\
{\left[Z_{k+1},+Z_{k+2}\right]=Z_{k+1}+\sum_{l=0}^{k-1} D^{l} Z_{l}}
\end{gathered}
$$

hold true. If $Z_{k+1}=0, D^{l}=0$, the subalgebra is trivial: one add operator $Y_{1}$ to subalgebras in $L_{12} \oplus\left\{Y_{p}\right\}$.

The rule of forming the optimal system for $L_{12} \oplus\left\{Y_{1}, Y_{p}\right\}$ is as follows. For a subalgebra in $L_{12}$ we choose we three basis operators including the operator containing $X_{12}$ so that the others form an ideal. Then for the chosen operators one should check identity (2.2).

Search of subalgebras in $L_{12}$ with such properties leads us to the following result.

If the subalgebra $\left\{Z_{1}, \ldots, Z_{k-1}, Z_{k+1}, Z_{k+2}\right\}$ is Abelian, then it is impossible to create a subalgebra in $L_{12} \oplus\left\{Y_{1}, Y_{p}\right\}$. It is surely, $Y_{1}$ is added to operator $X_{10}$ (or to $X_{n}, n=1,2$ ), at that $Z_{k+2}$ contains $X_{11}$, and operator with $X_{12}$ reads as $X_{12}-X_{11}+\ldots$ (or it does not contain $X_{11}$ ).

Looking through the optimal system in work [3], we single out the following subalgebras: $3.2(a=1, b=-1) ; 4.5,7(b=1), 7(b=0), 9,10,18 ; 5.5,7(a=1, b=-1), 8(a=1, b=-1), 12$, $17(a=1, b=-1), 21(a=1, b=0), 23,24(a=0), 25,33,34,35,36 ; 6.5,7,8,10,11,12,13(a=1, b=0)$, $17(\mathrm{a}=1, \mathrm{~b}=0), 18,19,20,20(\mathrm{a}=0) ; 7.5,6,8,9(\mathrm{a}=1, \mathrm{~b}=-1), 13(\mathrm{a}=1, \mathrm{~b}=0), 13(\mathrm{a}=1, \mathrm{~b}=-1), 15$, $16(a=0), 19(a=1, b=0), 20(a=0), 21,21(a=0) ; 8.5,7,9(a=1, b=0), 9(a=1, b=-1), 10,10(a=0)$, $11(\mathrm{a}=1, \mathrm{~b}=0), 12,16 ; 9.2,3,4(\mathrm{a}=1, \mathrm{~b}=0), 4(\mathrm{a}=1, \mathrm{~b}=-1), 5,6 ; 10.4$.

For instance, the subalgebras generated by 5.34 in [3] can be of two types $\left\{Y_{1}+X_{3}, a X_{1}+\right.$ $\left.X_{2}, X_{4}, Y_{p}+X_{11}, X_{12}\right\}$ and $\left\{X_{3}, Y_{1}+a X_{1}+X_{2}, X_{4}, Y_{p}+X_{11}, X_{12}\right\}$.

Each subalgebra in the algebra $L_{12} \oplus\left\{Y_{1}, Y_{p}, Y_{p^{2}}\right\}$ with a three-dimensional projection on $P_{3}$ is represented by the basis $Z_{1}=X_{i_{1}}+\ldots, \ldots, Z_{k}=X_{i_{k}}+\ldots, Y_{1}+Z_{k+1}, Y_{p}+Z_{k+2}, Y_{p^{2}}+Z_{k+3}$, where $Z_{k+1}, Z_{k+2}, Z_{k+3}$ and the dots stand for linear combination of basis elements in $L_{12}$ not involving $X_{i_{1}}, \ldots, X_{i_{k}}$. Arguments similar to that made in the end of Section 1 lead us to trivial extensions, i.e., to each subalgebra in $L_{12}$ one should add operators in $P_{3}$. 


\section{BIBLIOGRAPHY}

1. L.V. Ovsyannikov. The "PODMODELI" program. Gas dynamics // Prikl. matem. i mekh. 58:4, 30-55 (1994). [J. Appl. Math. Mech. 58:4, 601-627 (1994).]

2. S.V. Khabirov. Nonisomorphic Lie algebras admitted by gasdynamic models // Ufimskij matem. zhurn. 3:2, 87-90 (2011). [Ufa Math. J. 3:2, 85-88 (2011).]

3. E.V. Makarevich. Optimal system of subalgebras admitted by the gas dynamics equations in case of state equation with separated density // Siberian Math. Electr. Rep. 8, 19-38 (2011). (in Russian).

4. D.T. Siraeva. Optimal system of non-similar subalgebras of sum of two ideals // Ufimskij matem. zhurn. 6:1, 94-107 (2014). [Ufa Math. J. 6:1, 90-103 (2014).]

Salavat Valeevich Khabirov,

Institute of Mechanics USC RAS,

Oktyabr' av., 71,

450054, Ufa, Russia

E-mail: habirov@anrb.ru 